\title{
Daily medication use in nursing home residents with advanced dementia
}

\author{
Barbara Resnick ${ }^{\dagger 1}$ \& Elizabeth Galik ${ }^{1}$
}

Evaluation of: Tjia J, Rothman MR, Kiely DK et al.: Daily medication use in nursing home residents with advanced dementia. J. Am. Geriatr. Soc. 58, 880-888 (2010). The purpose of this study was to describe the pattern and factors associated with daily medication use in nursing home residents who have advanced dementia. This was a prospective cohort study and included 22 nursing homes in Boston (MA, USA). The participants were noted to receive an average of 5.9 medications daily $( \pm 3.0)$ and $37.5 \%$ received at least one medication that was 'never appropriate' for individuals with advanced dementia. Questionably beneficial medications were commonly used with those who have advanced dementia, even as individuals approached death. The authors recommended that medications were tailored for residents with advanced dementia so that they could be focused on optimizing the goals of care.

\section{Summary of methods \& results}

Overall, it is known that residents in nursing homes are prescribed approximately seven to eight medications daily $[1,2]$. The majority of the medications used in nursing homes treat chronic illnesses such as hypertension, diabetes or degenerative joint disease. Unfortunately, there are risks and benefits associated with all medications. For this reason, Tjia and her research team were particularly interested in examining drug use over the course of 18 months among residents with advanced dementia, as well as evaluating the use of drugs that are considered, based on expert consensus, 'never appropriate' for use in these individuals [3].

To achieve these objectives, a sample of 323 nursing residents with advanced dementia were recruited from 22 facilities in the Boston area (MA, USA) over a 3-year period. The mean age of participants was 85.3 years and the majority were female, white and had severe cognitive impairment. Advanced dementia was determined based on Minimum Data Set assessment and a Cognitive Performance Scale of 5 or 6. Additional eligibility criteria included being 60 years of age or older, living in the facility for at least 30 days, a Global Deterioration Scale score of 7 and a proxy who could speak English. The data collected included demographic information, cognitive disability was measured based on the Test for Severe Impairment, function was evaluated based on the Bedford Alzheimer's Nursing Severity Subscale, and healthcare utilization was captured (acute illnesses, hospitalizations, physician and nonphysician provider visits, referrals to hospice and end-of-life care preferences) via chart review.
Findings from this study demonstrated that the most prevalent comorbid condition experienced by participants was hypertension, followed by osteoporosis, coronary artery disease and diabetes. The majority of residents $(90 \%)$ maintained end-of-life preferences that reflected comfort care. Generally, the participants were prescribed, on average, 5.9 medications daily $( \pm 3.0)$ and $6.3( \pm 3.3)$ medications during the last week of life. The most common medications used were those associated with gastrointestinal symptoms, followed by analgesics, antidepressants, cardiovascular medications, hematological agents, osteoporosis medications, diuretics, antipsychotics, anticonvulsants and dementia medications. Over the course of the last year of life, there was a decline in the use of osteoporosis medications, as well as in the use of cholinesterase inhibitors and memantine very close to the time of death. Conversely, and appropriately, opioid use rose dramatically in the last year of life. Approximately $38 \%$ of the study participants were prescribed at least one never-appropriate medication (e.g., memantine and cholinesterase inhibitors) and a smaller percentage (12\%) used two or more never-appropriate medications (with lipid-lowering medications being the most common drug maintained and used).

When controlling for the center where the resident lived, having a hospice referral or an acute illness had the strongest association with taking more daily medications. Being male, having a shorter length of stay, better functional ability and diabetes were all associated with receiving a never-appropriate medication. Having a do-nothospitalize order was associated with a lower likelihood of receiving an inappropriate medication.

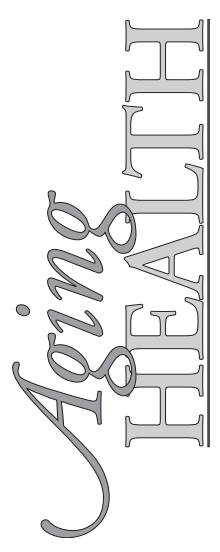

'University of Maryland School of Nursing, 655 West Lombard Street, Baltimore, MD 21201, USA

${ }^{+}$Author for correspondence: Tel.: +1 4107065178

Fax: +1 4107060344 resnick@son.umaryland.edu

\section{Keywords}

- Alzheimer's disease

- Beers criteria - comorbidities

- dementia $\bullet$ end of life

- inappropriate medication use

- long-term care • medication

- nursing home $\bullet$ polypharmacy

\section{future fenct $_{\text {medicine }}$ fsg}


The authors conclude that many nursing home residents with advanced dementia receive drug treatment that is no longer appropriate given their advanced disability and life expectancy. Obviously, the risk to the individual and the costs to the patient or society for what might be referred to as 'over-care' needs to be considered. However, one could make the case that, overall, the medication used with these individuals appears to be quite appropriate and simply focused on symptom management, with providers attempting to decrease the risk of discomfort associated with gastrointestinal symptoms, optimize mood and function, and treat pain. It is also possible that cardiovascular medications may have helped symptoms associated with atrial fibrillation. Without knowing the intent of the prescriber in these scenarios, it is difficult to evaluate the appropriateness or lack of appropriateness of each treatment decision.

\section{Discussion}

The use of the drugs in the never-appropriate group during the last year of life falls within the realm of futile care, and it is logical and ethical to stop medications in which there will be no long-term benefit achieved. At this point in time there is no mandate that says we must stop all medication in residents with advanced dementia. Furthermore, there is limited, if any, evidence to guide our decisions and help providers feel comfortable that there will be no deleterious effect associated with stopping a medication in this group. In retrospect, we can look at the last year of life, but, prospectively, it is increasingly difficult to determine when and what will be the last year of life, even among those with dementia $[4,5]$.

Information regarding the providers, family/resident perspectives and resident symptoms that may or may not have driven treatment is missing from the data captured in this study. There is evidence, for example, that memantine may help decrease behavioral symptoms in residents with moderate-to-severe dementia [6], and has a more favorable side-effect profile than antipsychotics and, thus, stopping the medication may have deleterious effects on the resident. The placebo effect is strong among families/proxies and it may be perceived that a treatment, such as a proton pump inhibitor and blood pressure medication, is critical to the quality of life of the resident.

\section{Future perspective}

In 1986, an Institute of Medicine report detailed the abuse, neglect and substandard care that was being received by nursing home residents in the USA [6]. This led to the Omnibus Budget Reconciliation Act of 1987 and, with it, many regulations regarding prescribing practices. In particular, there is a mandate that the resident should not receive any unnecessary drugs, with unnecessary being that without an adequate indication for use. Until 2004, there were 24 quality indicators, three of which were focused on psychotropic drug use. However, more recently, the quality indicators have been revised to encourage treatment of depression and pain. While this is all logical, it is hard to make drug decisions for individuals based on mandates and/or lists of medication deemed inappropriate for use according to the Beers criteria, or those deemed 'never appropriate' in advanced dementia based on consensus. In fact, it is with this group of individuals that we need to address symptoms individually, speedily and in a way in which the symptoms will quickly dissipate.

The authors of this paper provide wonderful suggestions for how we should proceed with care in the future. Their results, they note, reinforce the need for shared decision-making between providers and families/proxies of residents with severe dementia so that the goals of care are clear to all. In addition, they suggest reviewing the advantages and disadvantages of each medication, and making sure that the medication being used will help to achieve resident goals of care. These are wise recommendations and we would add simply that future research with this ever-growing group of residents needs

\section{Executive summary}

- Residents in nursing homes are commonly prescribed, on average, six daily medications.

- A total of $37 \%$ of residents receive at least one medication considered 'never appropriate' for individuals with advanced dementia.

- Residents who were older, male, not white, had non-Alzheimer's dementia, better cognition, cardiovascular disease, acute illness and who had been referred to a hospice were all associated with increased use of medications in general.

- Residents who were male, had shorter lengths of stay in the nursing home, better functional status and were diabetic were more likely to receive inappropriate medications.

- Ideally, medication use for nursing home residents with advanced dementia should be tailored to care-related goals. 
to focus on the impact of medication use with comfort (e.g., freedom of symptoms) as an outcome. A bold study in which futile medications are discontinued and residents monitored needs to be performed. We have tended to use 'quality of life' as a proxy for a good outcome in some studies of nursing home residents, but what we are really addressing with regard to medication use and management should be driven by symptom management. Do medications directly or indirectly influence pain, constipation, indigestion, anxiety, apathy, anorexia or dizziness? In addition, research needs to demonstrate the effectiveness of behavioral interventions and the use of technology to manage symptoms, and to test combined drug and behavioral interventions to optimize outcomes.

\section{Financial \& competing interests disclosure}

The authors have no relevant affiliations or financial involvement with any organization or entity with a financial interest in or financial conflict with the subject matter or materials discussed in the manuscript. This includes employment, consultancies, honoraria, stock ownership or options, expert testimony, grants or patents received or pending, or royalties.

No writing assistance was utilized in the production of this manuscript.

\section{Bibliography}

1. Gruber-Baldini A, Stuart B, Zuckerman I, Simoni-Wastila L, Miller R: Treatment of dementia in community-dwelling and institutionalized medicare beneficiaries. J. Am. Geriatr. Soc. 55, 1508-1516 (2007).

2. Holmes H, Sachs G, Shega J, Hougham G, Cox HD, Dale W: Integrating palliative medicine into the care of persons with advanced dementia: identifying appropriate medication use. J. Am. Geriatr. Soc. 56, 1306-1311 (2008).
3. Kruse RL, Oliver D, Mehr DR, Petroski GF, Swenson DL, Zweig SC: Using mortality risk scores for long-term prognosis of nursing home residents: caution is recommended. J. Gerontol. A Biol. Sci. Med. Sci. 65(11), 1235-1241 (2010).

4. Mitchell SL, Kiely DK, Hamel MB, Park PS, Morris JN, Fries BE: Estimating prognosis for nursing home residents with advanced dementia. J. Am. Med. Assoc. 291, 2734-2740 (2004).
5. Cummings J, Schneider E, Tariot $P$, Graham S: Behavioral effects of memantine in Alzheimer disease patients receiving donepezil treatment. Neurology 67, 57-63 (2006).

6. Institute of Medicine Committee on Nursing Home Regulations: Improving the Quality of Care in Nursing Homes. National Academy Press, Washington DC, USA (1986). 